\title{
A Noise Predictive Maximum Likelihood Equalization and Its Application in High Speed Serial Link Systems
}

\author{
Yinhang Zhang, Qingsheng $\mathrm{Hu}^{+}$and Jun Feng \\ Institute of RF- \& OE-ICs Southeast University, Nanjing, 210096, China
}

\begin{abstract}
This paper presents noise predictive maximum likelihood (NPML) detection for high-speed electrical backplane channels. Different from the partial response maximum likelihood (PRML) whose optimum coefficients are obtained from optimal decision feedback equalization (DFE), NPML achieves its coefficients by a noise prediction method which can be further incorporated with the computation of branch metrics in Viterbi algorithm, resulting in the removing of extra DFE and the simplification of hardware realization. The simulation results show that NPML detection can obtain better detection gain over conventional DFE when compared with PRML detection.
\end{abstract}

Keywords: backplane; noise predictive; decision feedback equalization

\section{Introduction}

Along with the growth of data transmission speed, the frequency-dependent loss characteristics and cochannel interference of legacy backplane cause the overlap of adjacent signals, i.e., inter-symbol interference(ISI), which may result in the eye diagram of received signal closed and the bit error rate (BER) of the overall backplane links deteriorated. One useful approach to combat channel characteristics is to use various equalization strategies [1-2]. The most commonly used equalization for high-speed backplane links is nonlinear decision feedback equalization (DFE). The traditional symbol-by-symbol (SSD) based DFE, however, has an unavoidable problem named error propagation which may worsen the BER performance. Different from DFE, which attempts to equalize the signal to remove as much of the ISI as possible, a PR maximum likelihood sequence detection (PRMLSD) aims to equalize the received signal to a known PR response can produce better performance without error propagation by combining with a maximumlikelihood such as Viterbi algorithm. To simplify the hardware realization of PRMLSD, this paper researches a noise-predictive PRMLSD and compares it with the conventional DFE.

\section{DFE and PR Equalization}

\subsection{DFE}

Fig. 1 is the architecture of traditional DFE which consists of a feed-forward filter, which is used to cancel pre-cursor ISI, and a feedback filter, mainly used to remove post-cursor ISI. The output of DFE equalizer can be given by:

$$
\mathrm{y}(n)=\sum_{i=1}^{0} a_{z} z(n-i)-\sum_{k=1}^{N} b_{k} d(n-k)
$$

where $z(n)$ is the input of equalizer, $d(n)$ is the output of SSD, $a_{i}(\mathrm{i}=-L \sim 0)$ and $b_{k}(k=1 \sim N)$ are the coefficients of feed-forward and feedback filter, respectively. Usually, $a_{i}$ and $b_{k}$ can be found by the channel impulse response. Fig. 2 is an example of channel impulse response, in which the solid line is the input signal and the

${ }^{+}$Corresponding author. Tel.: + 8615850510863.

E-mail address: qshu@ seu.edu.cn. 
dotted line is the impulse response. We can see that after being transmitted through the loss channel, the ideal pulse is attenuated and broadened due to ISI.

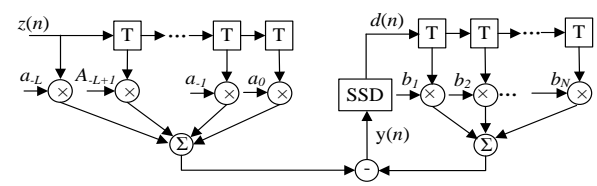

Fig. 1: A typical DFE architecture

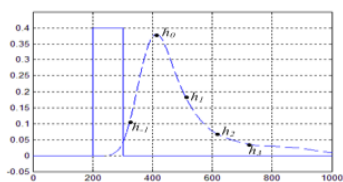

Fig. 2: Channel impulse response

\subsection{PRMLSD}

With the aim of equalizing the received signal to a known PR response, PRMLSD is different from the conventional DFE. Fig. 3 shows the architecture of PRMLSD, which consists of two blocks: a PR filter and a Viterbi algorithm module, mainly concerns the operations such as add-compare-select (ACS) and trace back (TB). PR filter can be expressed by:

$$
\hat{z}(n)=\sum_{l=0}^{N} h(l) a(n-l)
$$

where $\hat{z}(n)$ is the output of an ideal partial response at time $n, a(n)$ is the reference data in Fig.4, $h(l)$ is the coefficient of PR filter, and $l=0$ denotes the current symbol. The coefficient of PR filter $h(l)$ should be as accurate as possible in order to obtain good equalization performance.

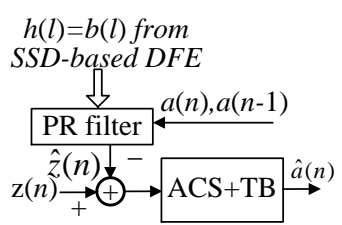

Fig. 3: PRMLSD

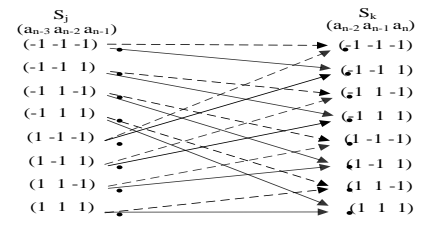

Fig. 4: An eight-state trellis diagram

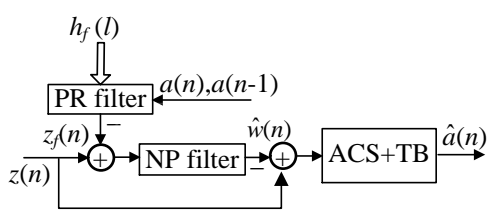

Fig. 5: NPMLSD

As for the second block, by Viterbi algorithm the detector can determine the most-likely transmitted sequence from all of the transmitted sequence to raise the performance of PR equalization [3-4]. Fig.4 gives an 8-state trellis diagram of Viterbi algorithm. The branch metric between two states $j$ and $k$ at time $n$ is the Euclidean distance between the received equalized symbol sample and the known partial response signals and can be calculated by

$$
b m(j, k, n)=\left[z(n)-\sum_{l=0}^{N} h(l) a(n-l)\right]^{2}
$$

And for state $j$, the state metrics which are accumulated by branch metrics at time $n$ can be described by:

$$
\operatorname{sm}(j, n)=\min [\operatorname{sm}(k, n-1)+b m(j, k, n), s m(l, n-1)+b m(j, l, n)]
$$

where $k$ and $l$ represent two possible states from which state $j$ can be arrived at. Actually, Eq. (5) responds to the operation of so called add-compare-select. Finally, the best path can be selected by a trace back operation. The most-likely transmitted sequence is the sequence with the least accumulated state metrics.

\subsection{Noise predictive MLSD}

As mentioned above, by using optimized $h(l)$ and Viterbi algorithm, PRMLSD can outperform conventional DFE at the cost of additional DFE filter although it is not always necessary. To remove the expansive DFE while obtain desired performance, a noise predictive MLSD (NPMLSD) is explored in this paper. Suppose $\hat{z}(n)$ is the samples expected for the data at time $n$, then from Eq. (2), we have:

$$
\hat{z}(n)=z_{f}(n)+w(n)=\sum_{l=0}^{N} h_{f}(l) a(n-l)+w(n)
$$

where $h_{f}(l)$ denotes a fixed PR coefficient which can be set based on the experience and $w(n)$ is the total distortion including noise component and residual interference. It is obvious that the more accurately $w(n)$ is estimated, the more residual interference components or noise can be eliminated. So, NPMLSD should 
include a NP filter as well as a PR filter and a Viterbi algorithm module, shown as Fig.5, in which $\hat{w}(n)$ is the estimation of $w(n)$ and NP filter adapts its coefficients with least mean square (LMS) algorithm.

Below we will illustrate how NPMLSD works. First we define $\hat{w}(n)$ :

$$
\hat{w}(n)=\sum_{i=1}^{K} p_{i} w(n-i)
$$

where $p_{i}(i=1 \sim K)$ is the coefficient of NP filter. Actually the adaptive predictor coefficients can be obtained offline using LMS algorithm whereby the coefficients are adapted per Eq. (8). $\mu$ is the adaptation gain and $e(n)$ is the error of predictive noise at time $n$. The equalized sample can be obtained by Eq. (10)

$$
\begin{gathered}
p_{i}(n+1)=p_{i}(n)-\mu e(n) w(n-i) \\
e(n)=w(n)-\hat{w}(n) \\
\hat{z}(n)=\sum_{l=0}^{N} h_{f}(l) a(n-l)+\sum_{i=1}^{K} p_{i} w(n-i)
\end{gathered}
$$

Furthermore, the result of noise estimation can be incorporated directly into the branch metrics of Eq. (4):

$$
\begin{aligned}
b m(j, k, n) & =\left[z(n)-\sum_{l=0}^{N} h_{f}(l) a(n-l)-\sum_{i=1}^{k} p_{i} w(n-i)\right]^{2} \\
w(n-i) & =z(n-i)-\sum_{l=0}^{N} h_{f}(l) a(n-i-l)
\end{aligned}
$$

And from Eq. (6) $w(n-i)$ can be expressed by Eq. (12). Finally, by substitute Eq.(12) into Eq. (11) the branch metrics which is only dependent on the input data $a$ and the known NP filter is obtained. Take PR2 response and 2-tap NP filter as an example where $N=1$ and $K=2$, the corresponding branch metrics is:

$b m(j, k, n)=\left[z(n)-h_{f}(0) a(n)-h_{f}(1) a(n-1)-p_{1}\left[z(n-1)-h_{f}(0) a(n-1)-h_{f}(1) a(n-2)\right]-p_{2}\left[z(n-2)-h_{f}(0) a(n-2)-h_{f}(1) a(n-3)\right]\right]^{2}$

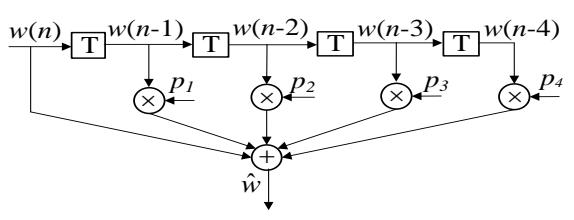

(a) A 4-tap NP filter

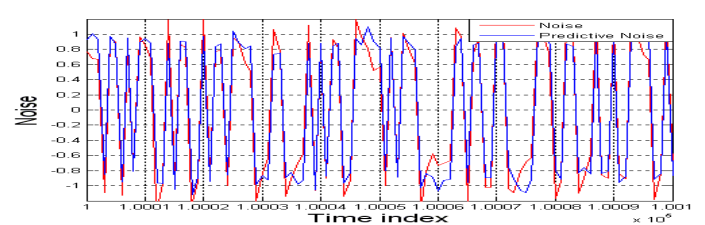

(b) Simulation result of 4-tap NP filter

Fig. 6: NP filter

It illustrates that NPMLSD can be realized with easy by incorporating the noise prediction with the computation of branch metrics in Viterbi algorithm while does not need an extra DFE. From Eq. (12) it can also be observed that an NP filter with more taps will benefit the performance of NPMLSD. Fig. 6 shows a 4-tap NP filter and its simulation result, from which we can see that the predictive noise is very close to the practical one.

\section{Simulation Experiments}

Fig. 7 gives our simulation platform in which 3 kinds of equalization scheme are simulated: DFE, PRMLSD and NPMLSD. In order to analyze and compare the performance of DFE and other two PR equalization schemes, all the performances are evaluated in terms of BER. Fig. 8 shows the channel magnitude response of four channels. And the insertion loss at $6.25 \mathrm{GHz}$ and $12.5 \mathrm{GHz}$ are shown in Table 1 . Table 2 gives the main parameters used in the simulation.

\subsection{Results analysis}

Fig.9 gives the simulated result of eye diagram for SSD-based DFE. Fig. 10 gives the output BER versus input SNR for the three equalization techniques. In the legends DFE3SSD means 3-tap SDD based DFE. DFE3MLSD means PRMLSD equalization with PR coefficients obtained from optimized DFE tap coefficients through adaption for a 3-tap DFE. PR2NP2MLSD and PR2NP4MLSD refer to NPMLSD 
detection with a PR2 partial response followed a NP filter, the only difference between them is the tap number: the former is 2 , and the latter is 4 . We can observe that for the lower loss channel A with a loss of $7.5 \mathrm{~dB}$, the detection gain of PR2NP2MLSD is around $1.2 \mathrm{~dB}$ at $10^{-6} \mathrm{BER}$, not significant. For PR2NP4MLSD, however, the gain is increased to $2 \mathrm{~dB}$. For channel $\mathrm{D}$, the detection gain of NPMLSD can be up to $2.5 \mathrm{~dB}$ at $10^{-3} \mathrm{BER}$, while it is only $2 \mathrm{~dB}$ for PR2NP2MLSD. It can be summarized that for all of the channels, MLSD based detections have detection gains over conventional DFE and PR2NP4MLSD has the highest one.

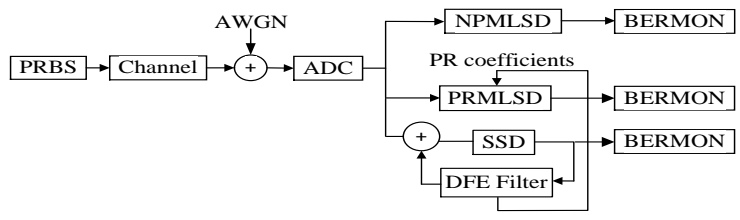

Fig. 7: Simulation platform

Table 1: Channel insertion loss

\begin{tabular}{|c|c|c|}
\hline \multirow{2}{*}{ Channel } & \multicolumn{2}{|c|}{ Insertion loss(dB) } \\
\cline { 2 - 3 } & $6.25 \mathrm{GHz}$ & $12.5 \mathrm{GHz}$ \\
\hline A & 4.557 & 7.561 \\
\hline B & 7.036 & 10.874 \\
\hline C & 11.617 & 19.007 \\
\hline D & 12.356 & 24.882 \\
\hline
\end{tabular}

Fig. 8: Channel magnitude response

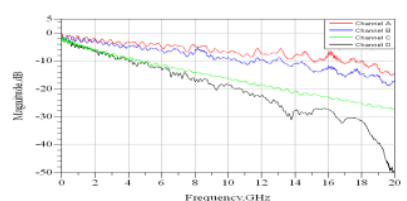

\begin{tabular}{|c|c|}
\hline Modulation & NRZ \\
\hline Symbol rate & 25Gb/s \\
\hline Test pattern & PRBS31 \\
\hline State number & 8 \\
\hline Branch metrics & 16 \\
\hline Simulation results & Eye, BER curve \\
\hline Path memory length & 48 \\
\hline Number of DFE taps & 3 \\
\hline Analysis mode & Statistical \\
\hline
\end{tabular}

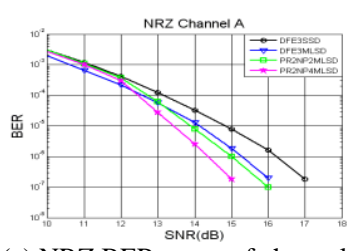

(a) NRZ BER curve of channel A

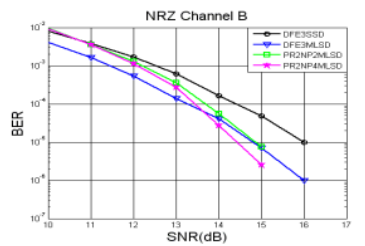

(b) NRZ BER curve of channel B

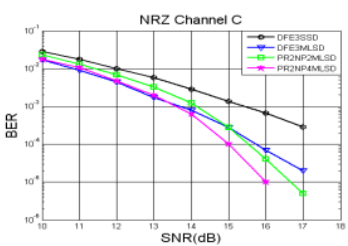

(c) NRZ BER curve of channel C

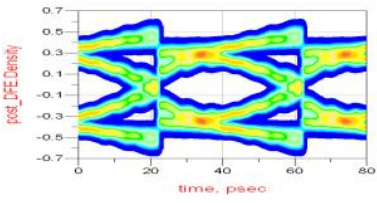

Fig. 9: Eye simulation

Fig. 10: BER simulation results

\section{Conclusion}

In this paper, noise predictive maximum likelihood equalization, partial maximum likelihood equalization and decision feedback equalization are discussed and analyzed in detail. The simulation results show that NPML detection can achieve better detection gain over conventional DFE when compared with PRML detection by using noise prediction method and Viterbi algorithm, while need not extra DFE, consequently, NPML equalization will be worth considering for high-speed backplane system for improving signal integrity.

\section{References}

[1] Z. Mingke, H. Qingsheng. A $6.25 \mathrm{~Gb} / \mathrm{s}$ equalizer in $0.18 \mu \mathrm{m}$ CMOS technology for high-speed SerDes, semiconductors, 2013, 34(12): 125010-1 - 125010-7.

[2] P. Amleshi, Z Yang, C Zhong. Design optimization study of 25Gbps electrical backplane links. Proc. of DesignCon2011, 2011, pp. 1750-1781.

[3] E. Eleftheriou, R. Hutchins. Adaptive noise-predictive maximum-likelihood (NPML) data detection for magnetic tape storage systems, IBM Journal of Research and Development, 2010, 54 (2): 7:1-7:10.

[4] L. Joo, L. Jae. A simplified noise predictive partial response maximum likelihood detection using M-algorithm for perpendicular magnetic recording channels. IEEE Transactions on Magnetics, 2005, 41(2):1064-1066. 